\title{
Transcatheter Technologies in Treating Brain Atherosclerosis Complicated by Chronic Cerebrovascular Insufficiency
}

\author{
Ivan V. Maksimovich \\ Clinic of Cardiovascular Diseases Named after Most Holy John Tobolsky, Moscow, Russia \\ Email: carvasc@yandex.ru
}

Received 22 June 2014; revised 21 July 2014; accepted 19 August 2014

Copyright (C) 2014 by author and Scientific Research Publishing Inc.

This work is licensed under the Creative Commons Attribution International License (CC BY).

http://creativecommons.org/licenses/by/4.0/

(c) (i) Open Access

\section{Abstract}

The research investigates the possibility of restoring the blood supply in patients with atherosclerosis of the brain, as well as the treatment of chronic cerebrovascular insufficiency. The research involves 946 patients aged 29 - 81 (average age 74) suffering from various types of cerebral atherosclerosis. $568(60.04 \%)$ patients underwent transcatheter treatment-Test Group. 378 $(39.96 \%)$ patients underwent conservative treatment-Control Group. The examination plan included laboratory diagnostics, assessment of dementia severity (CDR), assessment of cognitive impairment (MMSE), evaluation of the activities of daily living (IB), cerebral computed tomography (CT), cerebral magnetic resonance imaging (MRI), cerebral scintigraphy (SG), rheoencephalography (REG), cerebral multi-gated angiography (MUGA). To restore the blood supply, the method of transcatheter laser revascularization was applied; high-energy pulsed lasers were used for major intracranial arteries treatment, and low energy CW lasers-for distal intracranial branches treatment. Test Group: $459(80.81 \%)$ patients had good clinical outcome, $91(16.02 \%)$-satisfactory clinical outcome, $18(3.17 \%)$-relatively satisfactory clinical outcome; relatively positive clinical outcome was not obtained in any case. Control Group: good clinical outcome was not obtained in any case; $65(17.20 \%)$ patients had satisfactory clinical outcome, $121(23.26 \%)$-relatively satisfactory clinical outcome, $192(50.79 \%)$-relatively positive clinical outcome. The method of transcatheter laser revascularization of cerebral vessels is a physiological, effective and low-invasive treatment for patients suffering from atherosclerosis of the brain. The obtained results last up to 10 years and more; it causes regression of mental and motor disorders, promotes regression of dementia and largely improves the patients' quality of life; it has virtually no alternativewhich makes the proposed method significantly different from conservative treatment methods.

\section{Keywords}

Cerebrovascular Disease, Cerebrovascular Insufficiency, Cognitive Impairment, Transcatheter Laser Revascularization, Regeneration, Ischemic Stroke 


\section{Introduction}

Cerebrovascular atherosclerosis leads to chronic cerebrovascular insufficiency, progressive cerebral ischemia, which in turn cause progression of mental, cognitive, motor impairment and ultimately lead to stroke, disability and death [1]-[3]. Annual stroke mortality in the US is about 800,000 [4].

The brain is the most highly perfused organ; it is the first in the body to be blood supplied, and therefore its tissues are very sensitive to blood supply decrease [5]. Despite the fact that brain angioarchitectonics is varied and complex, it has been believed for a long time that atherosclerotic lesion of major extra- and intra-cranial branches leads to ischemic disorders [6] [7]. The importance of major branches is definitely high—but they perform the transport function. Slowly progressing occlusions of two or even three extracranial vessels turn on compensatory abilities - often progress without obvious symptoms and do not lead to fatal outcomes [2]. Under normal conditions, intracranial arterial and capillary bed is particularly important in the blood circulation and metabolism of cerebral tissue [2] [6]. A healthy human brain has 3 - 4 thousand capillaries in one cubic centimeter of brain tissue [5]. Ischemic processes stimulate angiogenesis and reorganization of cerebral circulation; the collateral bed, which makes blood supply to ischemic areas possible, becomes of great importance, while collateral branches have a capillary network of their own [2] [8]. With cerebral ischemia, the quickness of angiogenesis and the time when the collateral bed begins to function are very important [9].

Most often, the progression of cerebrovascular atherosclerosis is sufficiently slow; it is usually wide-spread affecting many minor arterial branches [2]. The process causes long chronic ischemia resulting in slowly progressing neurodegenerative disorders accompanied by atrophic tissue changes and growing mental and cognitive impairment [2] [8] [9]. This process is often accompanied by multiple scattered lacunar micro-strokes that progress unnoticeably enough, without marked ischemic attacks. During CT and MRI, these micro-strokes are not always detected due to their small size that is close to the resolution capabilities of the apparatus used.

Conservative treatment of such disorders can be effective only at the early stages of the disease, when stenotic lesions are not pronounced and widespread. With aggravating atherosclerotic lesions, patients require reconstructive or transcatheter surgery [10].

There has recently been appearing an increasing number of works dedicated to successful application of transcranial laser radiation in the treatment of the effects of the ischemic stroke [11] [12], cranial trauma and dementia [13]-[15]. The authors underline the good neuroprotective effect of the treatment contributing to successful neurorehabilitation, but it should as well be noted that the treatment is also effective at mild, early disease stages.

Reconstructive vascular surgery has worked well on extracranial vessels [16] [17]. Intracranial lesions make conducting such interventions rather difficult due to the anatomical features and the small diameter of the vessels. Reconstructive surgery aimed at creating extra-intracranial arterial anastomosis is not always effective, and there have recently been arguments concerning the feasibility of these interventions [18].

Interventional methods, such as balloon angioplasty and stenting of different types, have worked well on extracranial pabrachiocephalic branches [17] [19]. These interventions are often performed on intracranial arteries in acute conditions and are limited only by the proximal parts of the vascular bed [20]-[22]. Conducting interventions in the distal parts of a small diameter is of certain complexity.

Cerebral ischemia is definitely a multivalent concept; an important role is played in its progression by hypertension, hyperlipidemia, diabetes, disorders of systemic hemodynamics, microembolia caused by cardiac arrhythmias [23] [24], but in any situation they are rather secondary to the severe atherosclerotic lesion.

In the current situation, there remains a need to develop new integrated transcatheter treatments.

The present research investigates the possibility of restoring cerebral perfusion in patients with atherosclerosis of the brain, both uncomplicated and complicated by micro-strokes, by means of transcatheter laser revascularization. The research also explores the possibility of reducing mental disorders and reconstructing intellectual and motor functions in these patients in the early and late postoperative period.

\section{Materials and Methods}

\subsection{Subject of Research}

All the studies and all transcatheter interventions have been carried out with the approval of the Ethics Committee, as well as with the consent of the examined and treated patients and their relatives. 
We examined 1238 patients aged from 29 to 81 (average age 74) suffering from various types of atherosclerotic lesions of cerebral vessels, of whom 916 were male (73.99\%) and 322-female (26.01\%) patients.

The examination plan included laboratory diagnostics, assessment of the severity of dementia (CDR), assessment of cognitive impairment (MMSE), evaluation of the activities of daily living (IB), cerebral computed tomography (CT), cerebral magnetic resonance imaging (MRI), cerebral scintigraphy (SG), rheoencephalography (REG), cerebral multi-gated angiography (MUGA).

\subsection{Examination of Patients}

Laboratory examination was performed according to the schemes generally accepted in iterventional neuroangiology and included clinical, biochemical and coagulologic tests.

CT and MRI of the brain were performed on "Somatom" (Siemens), "Hi Speed" (GE), "Tomoscan" (Philips), "Apetro Eterna" (Hitachi) following the classical method. Primary examination was conducted at the patient's admission and then at intervals of 6 - 12 months.

SG of the brain, with the determination of cerebral blood flow velocity, was carried out on a gamma camera (Ohio Nuclear, US) following the classical method in dynamic and static mode with TC 99M pertechnetat 555. Primary examination was conducted at the patient's admission and then at intervals of $6-12$ months.

REG was conducted by means of "Reospektr-8" (Neurosoft, Russia) in accordance with the standard automated method with the identification of pulse blood flow in the cerebral hemispheres. Primary examination was conducted at the patient's admission, secondary—before the patient's discharge and then at intervals of 6 - 12 months.

MUGA of the brain was performed on apparatus "Advantx" (GE) following the classical method of transfemoral access. Synchronously, taking into account the start and rate of administration, 10 - $12 \mathrm{ml}$ of Omnipack 350 was introduced intra-carotidally and 7 - $8 \mathrm{ml}$ intra-vertebrally. The registration was carried out in direct and side projections in constant subtraction mode at a speed of 25 frames per second. Further on, frame by frame analysis of the angiograms received in each phase contrast was conducted [25]. Capillary density contrast analysis was performed at the corresponding phase by means of an automatic method using computer program "Angio Vision" based on the determination of the degree of blackening of the corresponding part of the image [26].

Determination of the severity of dementia was made according to J.C. Morris's Clinical Dementia Rating scale (CDR) [27]. Primary examination was conducted either at the patient's first visit or on the admission day, secondary—before the patient's discharge and then at intervals of 6 - 12 months.

Assessment of cognitive impairment was conducted by means of Mini-Mental State Examination (MMSE) [28]. Primary examination was conducted either at the patient's first visit or on the admission day, secondarybefore the patient's discharge and then at intervals of $6-12$ months.

Evaluation of the activities of daily living was carried out with Index Bartels Functional Evaluation: the Barthel Index (IB) [29]. Primary examination was conducted at the patient's admission, secondary-before the patient's discharge and then at intervals of 6 - 12 months.

\subsection{Selection of Patients}

Criteria for selection of patients for this research:

1) consent of the patient and her/his relatives to conduct the examination and treatment;

2) somatic condition of patients that allowed to carry out the examination and treatment;

3) signs of chronic cerebrovascular insufficiency;

4) predominant atherosclerotic lesions of intracranial arterial branches.

For the present research, we selected 946 (76.41\%) patients aged from 29 to 81 (average age 74): 701 (74.10\%) male and 245 (25.90\%) female patients.

Transcatheter treatment was undergone by 568 (60.04\%) patients aged from 29 to 80 (average age 74), of whom 408 (71.83\%) were male and 160 (28.17\%)—female patients—Test Group.

Conservative treatment was undergone by 378 (39.96\%) patients aged from 33 to 81 (average age 75), of whom 259 (68.52\%) were male and 119 (31.48\%)—female patients—Control Group. This group consisted of patients for whom transcatheter treatment was unnecessary, or they and their relatives were not willing to undergo it. 


\subsection{Analysis of Patients}

The examination revealed the following disorders in patients selected:

- high blood lipids level was detected in $764(80.76 \%)$ cases;

- hypercoagulation was detected in 735 (77.70\%) cases;

- calcium deposits on the walls of cerebral blood vessels were detected in 775 (81.92\%) cases;

- general involutive changes of the cerebral cortex accompanied by subarachnoid space extension were detected in 758 (80.13\%) cases;

- expansion of Sylvian fissure was found in 612 (64.69\%) cases;

- reduction of the clearness of gray and white matter borders was found in 462 (48.83\%) cases;

- signs of unocclusive hydrocephalus were detected in 412 (43.55\%) cases;

- signs of leucoaraiosis were detected in 161 (17.02\%) cases;

- micro-focal (up to $2 \mathrm{~cm}$ ) postischemic brain cysts were detected in 132 (13.95\%) cases;

- single and multiple microcysts (up to 2 - $5 \mathrm{~mm}$ ) were detected in 116 (12.26\%) cases;

- absence of postischemic cysts against the background of atherosclerotic lesions was detected in 698 (73.78\%) cases (one cannot exclude the possibility of these patients having very small cysts-smaller than the resolution of the equipment used);

- reduced blood flow in the cerebral hemispheres was observed in all 946 (100\%) cases;

- reduced pulsed volume blood flow in the carotid and vertebrobasilar systems was detected in all 946 (100\%) cases;

- multiple atherosclerotic stenotic or occlusive lesions in the basin of the front, middle or posterior cerebral arteries were detected in all 946 (100\%) cases. Of these, two-sided lesion was detected in 912 (96.41\%) cases;

- signs of dementia equal to CDR-1 were detected in 264 (27.91\%) cases;

- signs of cognitive impairment (MMSE score below 28 points) were detected in 242 (25.58\%) cases;

- signs of reduction of the activities of daily living (IB below 100 points) were detected in 39 (4.12\%) cases.

In the long term (2 - 10 years), 482 (50.95\%) patients aged from 35 to 85 (mean age 76 years) were re-examined. Of these, Test Group patients comprised 286 (59.34\%), Control Group patients-196 (40.66\%).

CDR, MMSE, IB evaluation, as well as cerebral CT, MRI, SG and REG were performed in all cases, cerebral MUGA or MRA were performed in 72 (25.17\%) Test Group patients and in 34 (20.12\%) Control Group patients.

Test Group. Given the specificity of cerebral perfusion and cerebral angioarchitectonics, as well as the small diameter and tortuosity of the affected intracranial arterial branches, we applied the method of transluminal laser revascularization for the revascularization of the brain [25] [26] [30]. The method allows working on convoluted vessels of small diameter. Efficacy and safety of this method is proved in earlier experimental and clinical studies [2] [9] [30]-[34].

\section{The Method of Transcatheter Laser Revascularization of Cerebral Vessels}

High-energy impulse laser systems were used to conduct the revascularization of the brain with major intracranial arteries lesions; low-energy continuous laser systems were used for the revascularization of the brain with distal intracranial branches lesions [9] [25] [26] [30].

The essence of the method is the following:

Under local anesthesia, according to Seldinger's classical method, the common femoral artery is punctured and catheterized with an installation of an introducer with a diameter of 6-9F. Through this introducer and through coaxially brought guiding catheters installed in the general and further on in the internal carotid or in the vertebral artery, is brought a flexible fiber-optic instrument with a diameter of 50 to 200 micrometers, coupled with the laser unit. The fiber-optic instrument is guided to the site of intracerebral arteries lesion, after which the laser treatment is carried out. The distal end of the fiber-optic instrument is constantly washed with heparinized saline solution. Given the specificity of laser exposure on atherosclerotic tissue and the vascular wall [25] [26], the use of distal protection is not required. To carry out X-ray control, small doses of radiopaque substance are periodically introduced. The exposure time depends on the nature of the atherosclerotic lesion, the type of the laser system used, and is in general from 30 seconds to 40 minutes. After the endovascular intervention the patient undergoes repeated cerebral multi-gated angiography following the procedure described above, the results 
of which determine the degree of revascularization and restoration of the cerebral vascular bed [2] [30]. We assume that if the cerebral blood flow has not been fully restored during the first attempt of the intervention, the manipulation can be repeated, but it was not necessary during the transcatheter interventions described.

Taking into consideration the MUGA data and the fact that atherosclerosis is a systemic disease and cerebral vascular lesions progress in various basins, not just in the particular part of the bed, transcatheter interventions were usually carried out from two sides, in order to improve the blood supply of the whole brain. It allows for not only direct revascularization, but it also gives an opportunity to improve blood flow through the collateral bed from contralateral vascular basin [9].

The transcatheter treatment was followed by supportive therapy carried out by common interventional neuroangiology schemes and dosing. The patients underwent conventional desagrigant, anticoagulant, vasodilator and nootropic therapy including Aspirin, Heparin, indirect anticoagulants (depending on the blood coagulation indicators), Pentoxifylline $100 \mathrm{mg}$, Complamin $150 \mathrm{mg}$, Inosin $200 \mathrm{mg}$, Nootropil (Piracetam) $1200 \mathrm{mg}$ (or Gliatilin $1000 \mathrm{mg}$ ) intravenously, with a drop counter, NO 10 - 15, and then they took pills. Subsequent 1 - 2 months courses of pills treatment were repeated twice a year.

Control Group. Control Group patients underwent a therapeutic treatment following similar schemes and doses of the drugs. In the aftermath, they also took 1 - 2 months courses of infusion or pelleted drugs twice a year.

\subsection{Results Evaluation}

The results obtained were divided into the following groups:

- good clinical outcome after the treatment was considered to be almost complete restoration of mental and motor functions and activities of daily living;

- satisfactory clinical outcome was considered to be incomplete restoration of mental and motor functions and activities of daily living;

- relatively satisfactory clinical outcome was considered to be a partial recovery of mental and motor functions and activities of daily living;

- relatively positive clinical outcome was considered to be the absence of negative dynamics with a slight reduction of mental and motor functions and activities of daily living.

\section{Results}

\subsection{Test Group}

\subsubsection{Immediate Results}

A good immediate angiographic outcome manifested in the restoration of the lumen and patency of the vessels, as well as in collateral revascularization on the side with more pronounced atherosclerosis, was obtained in 546 (96.13\%) cases. Besides, improved blood supply in the cerebral hemispheres by means of the collateral revascularization from contralateral vascular basins was obtained in 542 (95.42\%) cases.

\subsubsection{SG and REG Results}

According to SG data, improved blood flow in the cerebral hemispheres, both on the more affected and on the contralateral side, was observed in all $568(100 \%)$ cases, and the positive trend remained during the whole observation period.

According to REG data, improved pulse-volume blood flow in the carotid and vertebrobasilar basins on the affected and contralateral sides was found in all 568 (100\%) cases, and the positive trend remained during the whole observation period.

\subsubsection{CT and MRI Data Results}

From the first 6 months after the transcatheter treatment, the patients tended to have diminishing general involutive changes of the cerebral cortex accompanied by narrowing of subarachnoid space.

In the subsequent period of 12 - 24 months:

- general involutive changes in the cerebral cortex decreased in 494 (95.92\%) of 515 cases;

- reduction of Sylvian fissure narrowing was observed in 384 (94.58\%) of 406 cases; 
- tendency to restore clearness of boundaries between gray and white matter of the brain was diagnosed in 204 (66.45\%) of 307 cases;

- reduction of signs of unocclusive hydrocephalus was detected in 178 (64.96\%) of 274 cases;

- signs of leucoaraiosis were detected in 99 (92.52\%) of 107 cases;

- signs of residual effects of micro-focal postischemic brain cysts were detected in 12 (13.64\%) of 88 cases;

- single or multiple microcysts were not identified in any case.

It should be noted that in a more distant period of time ( 2 - 10 years) the positive trend was maintained, which might indicate signs of cerebral tissue recovery.

\subsubsection{MUGA Results}

Repeated cerebral MUGA or MRA were performed at intervals of 2 - 10 years after the treatment in 72 (12.68\%) patients:

- preserved lumen and patency of the vessels, as well as inhensed collateral revascularization, were obtained in 69 (95.83\%) cases;

- preserved improved cerebral perfusion due to collateral revascularization from the contralateral basin was obtained in 67 (93.06\%) cases.

\subsubsection{Clinical Results}

Clinical results evaluation was conducted at various times after the transcatheter interference. The restoration of mental and motor functions depended largely on the severity of the initial ischemic disorders and on the timing of transcatheter intervention after the symptoms onset. The initial positive dynamics was observed quite soon after the transcatheter treatment. In the first 2 - 3 weeks after the conducted treatment, the patients' condition improved and mental and motor disorders decreased.

\section{1) Short-Term Clinical Results}

In 2 - 6 months after the treatment:

- good clinical outcome (almost full recovery of mental and motor functions) was obtained in 459 (80.81\%) cases;

- satisfactory clinical outcome (incomplete recovery of mental and motor functions) was obtained in 91 (16.02\%) cases;

- relatively satisfactory clinical outcome (partial recovery of mental and motor functions) was obtained in 18 (3.17\%) cases;

- relatively positive clinical outcome (no negative dynamics with a slight reduction of mental and motor functions) was not obtained in any case.

\section{2) Long-Term Clinical Results}

In the long-term period (2 - 10 years), 286 (62.31\%) patients were re-examined; the positive dynamics persisted in the vast majority of treated patients:

- good clinical outcome was initially obtained in 459 patients, of whom 228 (49.67\%) were re-examined; the result was preserved in 212 (92.98\%) cases;

- satisfactory clinical outcome was initially obtained in 91 patients, of whom 52 (57.14\%) were re-examined; the result was preserved in 48 (92.31\%) cases;

- relatively satisfactory clinical outcome was initially obtained in 18 patients, of whom 6 (33.33\%) were re-examined; the result was preserved in 5 (83.33\%) cases.

\section{3) Complications}

During transcatheter interventions, there was no thrombosis or embolism in the distal arterial bed; and there was no negative effect. 2 patients developed a minor hematoma at the puncture site of the femoral artery not requiring any surgical treatment, but this is not considered a complication of the treatment conducted.

Statistical analysis is presented in Table 1.

\subsubsection{Treatment Examples}

Patient H., 60 years old, female, suffered a small ischemic stroke in the right hemisphere resulting in partial left-sided hemiparesis; the patient notes decreased memory and vestibular disorders. In 2 months after the stroke, there are no signs of dementia, cognitive impairment level is equal to 26 MMSE points, the level of everyday life- to 90 IB points. CT of the brain revealed a moderate expansion of the subarachnoid space and a small hete- 
Table 1. Comparative characteristics of clinical results obtained after treatment of test and control group patients.

\begin{tabular}{|c|c|c|c|c|c|c|c|c|c|}
\hline \multirow[b]{2}{*}{ Category } & \multicolumn{5}{|c|}{ Aggregate results frequency table: short-term period } & \multicolumn{4}{|c|}{ Aggregate results frequency table: long-term period } \\
\hline & Group & Count & $\begin{array}{c}\text { Cumulative } \\
\text { count }\end{array}$ & Percent & $\begin{array}{l}\text { Cumulative } \\
\text { percent }\end{array}$ & Count & $\begin{array}{l}\text { Cumulative } \\
\text { Count }\end{array}$ & Percent & $\begin{array}{c}\text { Cumulative } \\
\text { percent }\end{array}$ \\
\hline $\begin{array}{l}\text { Relatively positive clinical } \\
\text { outcome }\end{array}$ & Test & 0 & 0 & 0 & 0 & 1 & 1 & 0.34965 & 0.3497 \\
\hline $\begin{array}{l}\text { Relatively satisfactory } \\
\text { clinical outcome }\end{array}$ & Test & 18 & 18 & 3.16901 & 3.1690 & 9 & 10 & 3.14685 & 3.4965 \\
\hline Satisfactory clinical outcome & Test & 91 & 109 & 16.02113 & 19.1901 & 64 & 74 & 22.37762 & 25.8741 \\
\hline Good clinical outcome & Test & 459 & 568 & 80.80986 & 100.0000 & 212 & 286 & 74.12587 & 100.0000 \\
\hline $\begin{array}{l}\text { Relatively positive clinical } \\
\text { outcome }\end{array}$ & Control & 192 & 192 & 50.79365 & 50.7937 & 144 & 144 & 73.46939 & 73.4694 \\
\hline $\begin{array}{l}\text { Relatively satisfactory } \\
\text { clinical outcome }\end{array}$ & Control & 121 & 313 & 32.01058 & 82.8042 & 38 & 182 & 19.38776 & 92.8571 \\
\hline Satisfactory clinical outcome & Control & 65 & 378 & 17.19577 & 100.0000 & 14 & 196 & 7.14286 & 100.0000 \\
\hline
\end{tabular}

The analysis of the results of the early and late periods of treatment within each group by means of the chi-square test revealed no significant differences between the initial treatment period and the results of the treatment at a later stage. To compare test and control groups at early and late stages of therapy, an analysis of contingency tables was conducted using the chi-square test. The compared groups differed significantly in the level of clinical effect at each stage of therapy ( $<<0.01$ ), and test group results were significantly better than control group results.

rogeneous post-ischemic cyst in the right hemisphere (Figure 1).

SG and REG showed quite a marked reduction in blood flow and pulse volume with the predominance of the process to the right.

Cerebral MUGA revealed multiple occlusions of the distal branches of the right middle cerebral artery, multiple diffuse stenosis of intracranial branches (Figure 2).

The patient underwent transcatheter laser revascularization first of the right and then of the left hemisphere. Postoperative cerebral MUGA revealed completely restored patency and lumen of the distal branches of the right middle cerebral artery and pronounced collateral revascularization on the two sides (Figure 3).

Repeated SG and REG showed positive dynamics of blood flow and pulse volume in both hemispheres of the brain.

A month later, the patient demonstrated the restoration of motor functions, intellectual abilities and professional skills. MMSE-30 points, IB-100 points; the patient returned to her professional activity.

In 12 months, repeated CT of the brain revealed restoration of subarachnoid space, reduction in the size of the post-ischemic cyst with signs of cerebral tissue structure recovery (Figure 4).

6 years after the transcatheter treatment, the patient does not have any complaints, there are no signs of dementia, no cognitive impairment, and she is actively engaged in intellectual activity, IB-100. CT of the brain revealed no signs of residual effects of the post-ischemic cyst; cerebral tissue structure of the right hemisphere is restored (Figure 5).

Cerebral MRA (Figure 6) showed: the patency and lumen of the distal branches of the right internal carotid artery are completely preserved; there is further progression of collateral revascularization.

\subsection{Control Group}

\subsubsection{Immediate Results}

Immediately after the course of conservative treatment, the patients had no negative dynamics, and there was certain positive dynamics manifested in diminishing mental and motor disorders.

\subsubsection{SG and REG Results}

SG and REG showed partial improvement of blood flow and pulse volume in the cerebral hemispheres in 253 (66.93\%) patients. The improvement was not stable, and subsequent examination in 12 - 36 months reported partial regression in 128 (50.60\%) cases. 


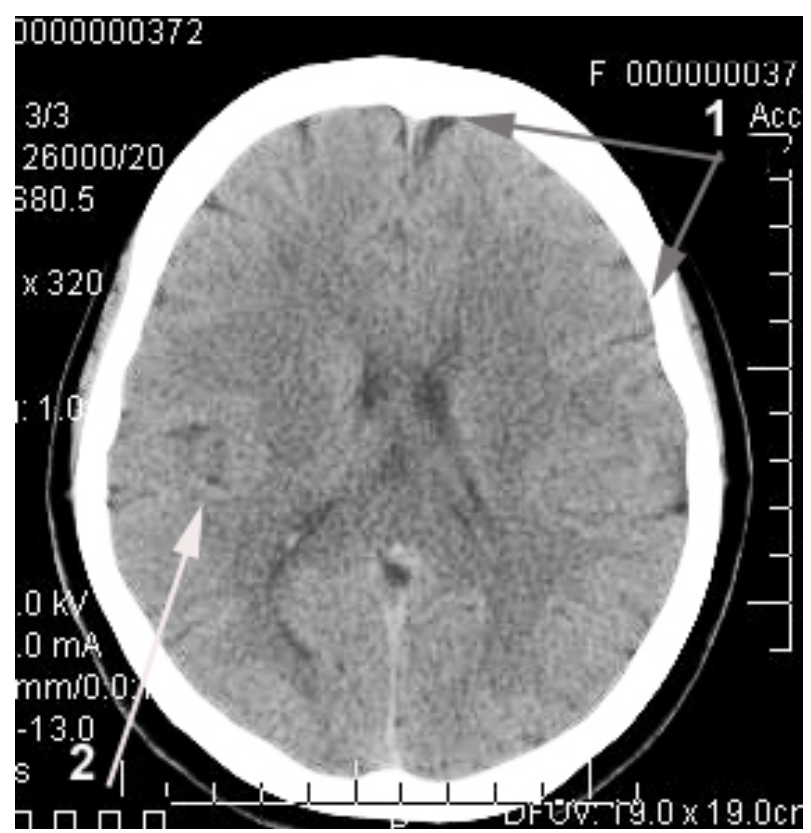

Figure 1. Patient H., female, 60 years old: CT of the brain. Before the intervention: 1) moderate expansion of the subarachnoid space; 2) moderate heterogeneous post-ischemic cyst in the right middle cerebral artery region.

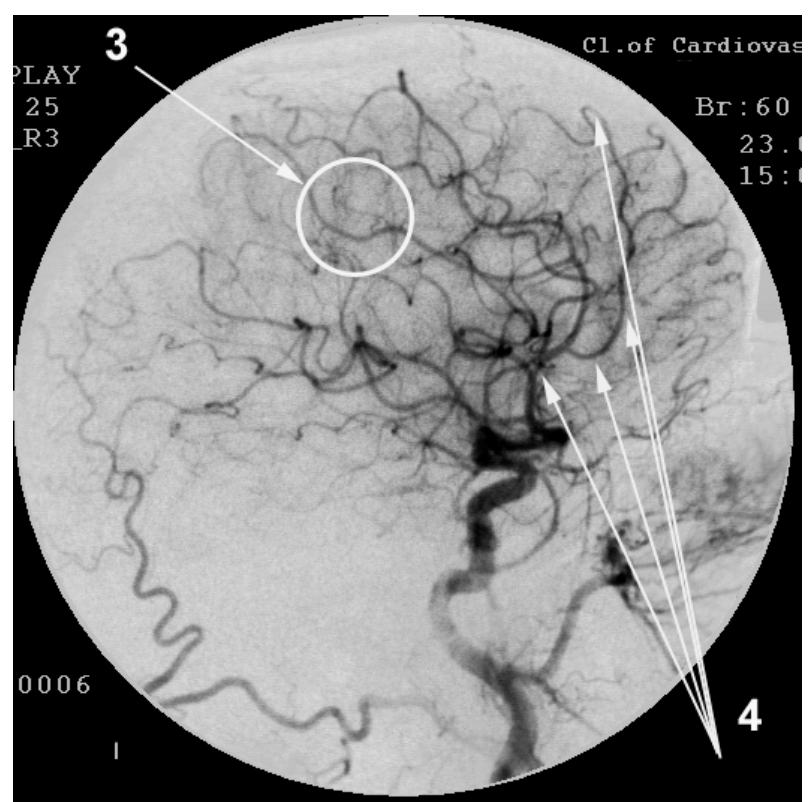

Figure 2. Same patient H.: right-sided carotid MUGA (lateral projection). Before the intervention: 3) occlusion of distal branches of the right middle cerebral artery; 4) multiple stenosis of intracranial branches.

\subsubsection{CT and MRI Results}

CT and MRI in the first 12 - 36 months after the treatment showed no substantial reduction of the involutive changes in the brain tissue in any of 243 patients. In the longer-term period, 137 (56.38\%) patients showed growing involutive changes. 


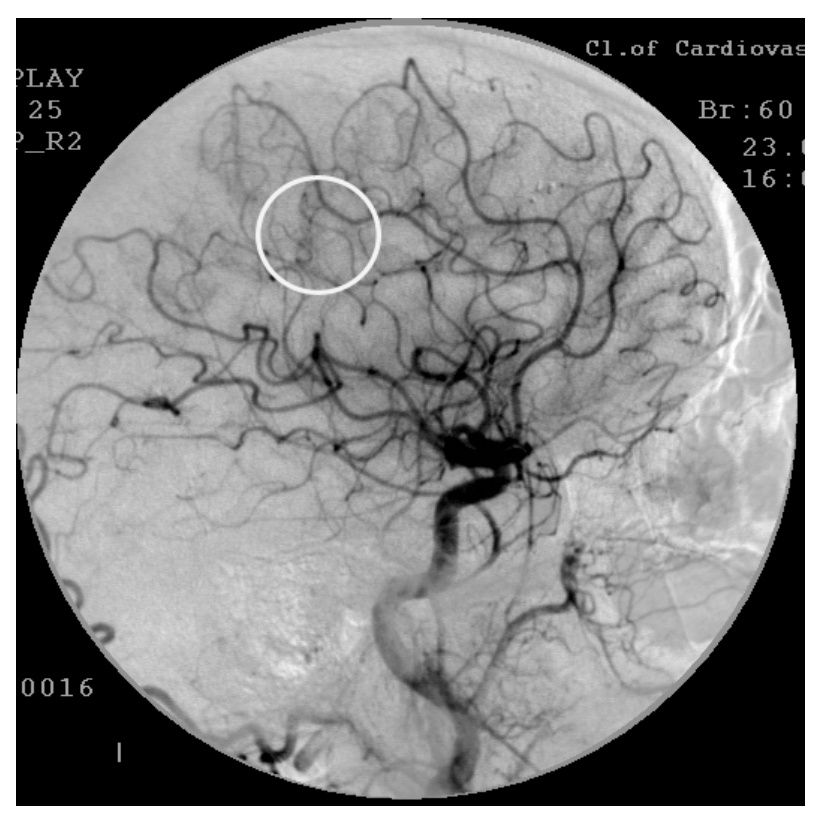

Figure 3. Same patient H.: right-sided carotid MUGA (lateral projection). After the intervention: restoration of the lumen and permeability of distal branches of the right middle cerebral artery, improvement of collateral blood flow.

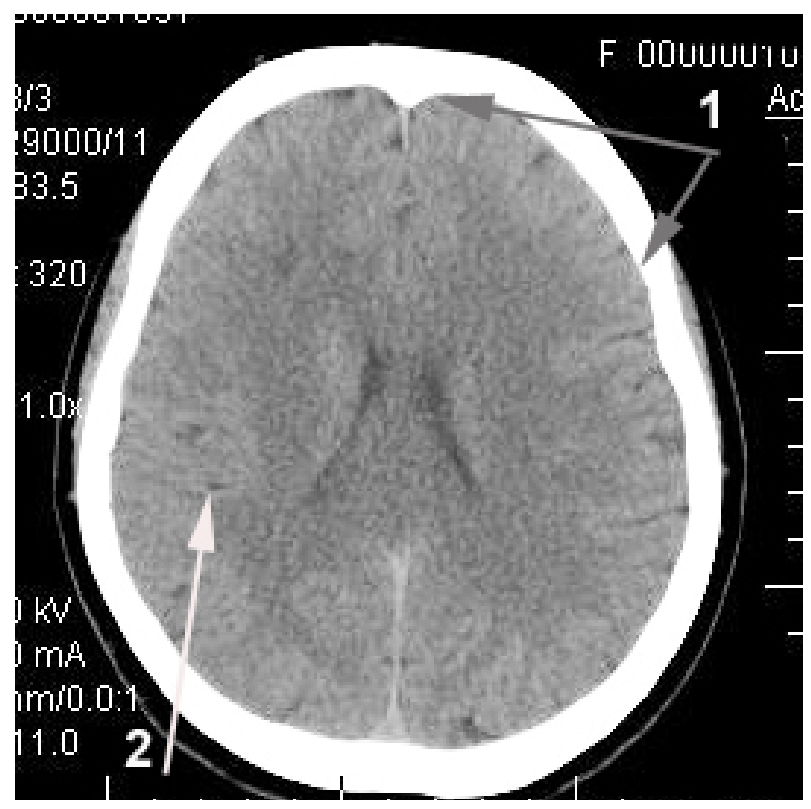

Figure 4. Same patient H.: CT of the brain. 12 months after the intervention: 1) subarachnoid space restoration; 2) reduction in the size of the post-ischemic cyst with signs of cerebral tissue structure recovery.

\subsubsection{MUGA Results}

Repeated cerebral MUGA or MRA were performed in $34(9.00 \%)$ cases at intervals of 2 - 10 years after the treatment started. There were no significant changes in the angioarchitectonics; 31 (91.18\%) patients had further progression of the atherosclerotic process. 


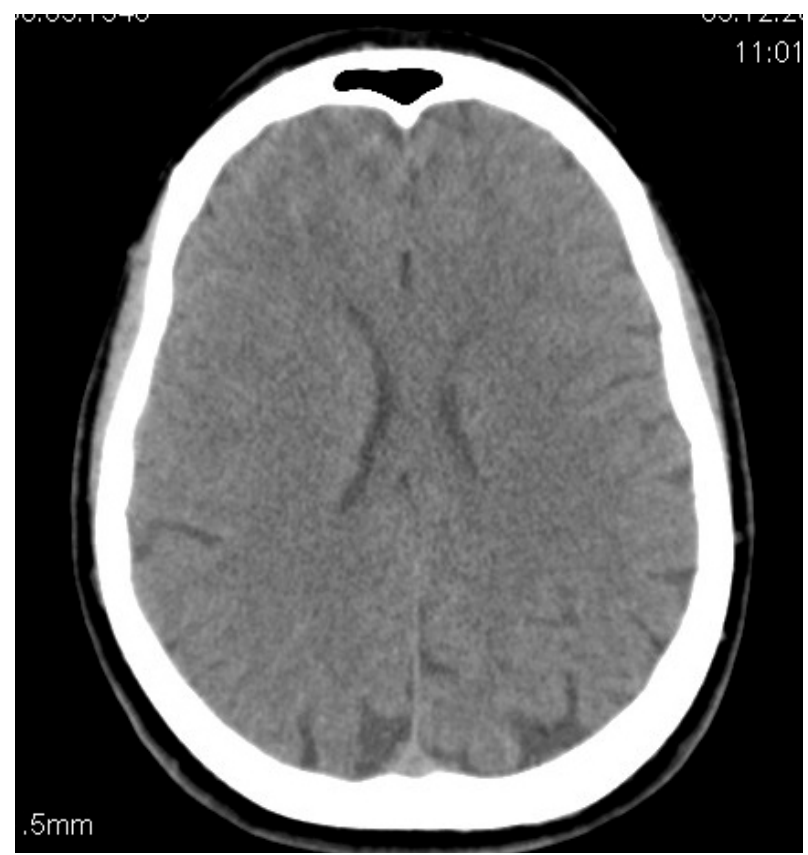

Figure 5. Same patient H.: CT of the brain. 6 years after the intervention: no signs of residual effects of the post-ischemic cyst, the structure of the right hemisphere cerebral tissue is restored.

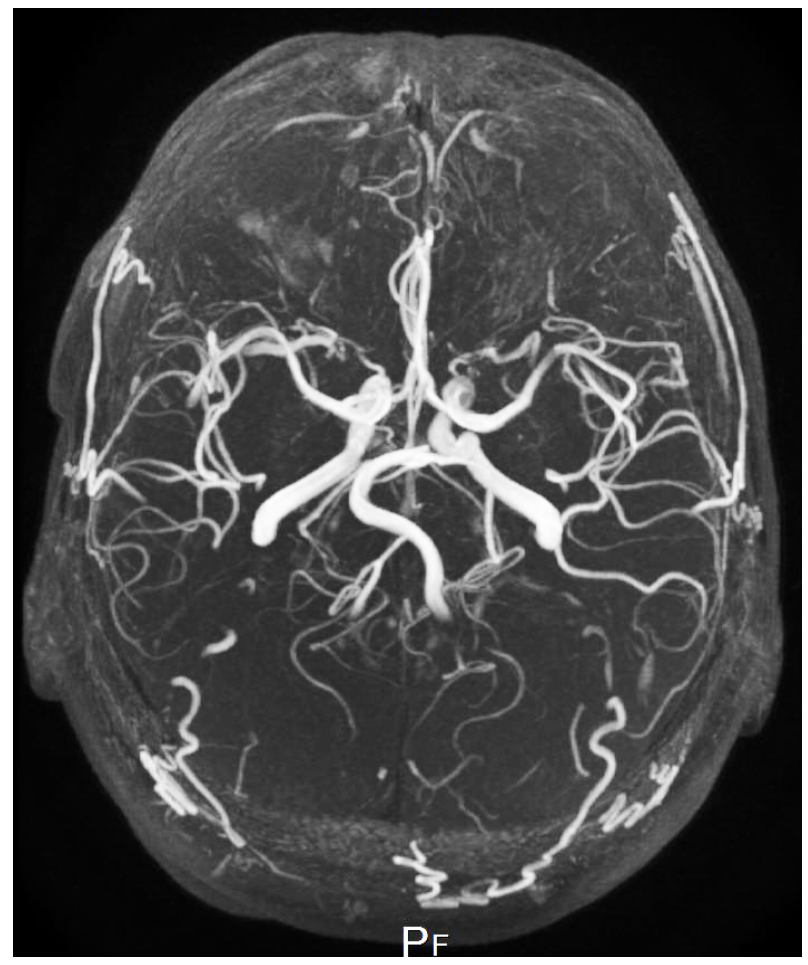

Figure 6. Same patient H.: MRA of the brain. 6 years after the intervention: the patency and lumen of the distal branches of the right internal carotid artery are completely preserved, there is further progression of collateral revascularization. 


\subsubsection{Short-Term (2 - 6 Months) and Long-Term (2 - 10 Years) Clinical Results}

- Good clinical outcome in the early period was not obtained in any case.

- Satisfactory clinical outcome in the early period was obtained in 65 (17.20\%) patients, of whom 36 patients were examined in the remote period; the result was preserved in 14 (38.88\%) cases.

- Relatively satisfactory clinical outcome in the early period was obtained in 121 (23.26\%) patients, of whom 74 were examined in the remote period; the result was preserved in 34 (45.95\%) cases.

- Relatively positive clinical outcome in the early period was obtained in 192 (50.79\%) patients, of whom 86 were examined in the remote period; the result was preserved in 40 (46.51\%) cases.

\subsubsection{Treatment Examples}

Patient K., 61 years old, male. Complaints of memory loss, intellectual impairment, periodic vestibular disorders; no specific signs of dementia, cognitive impairment equals 26 MMSE points, level of daily living-95 IB points. CT of the brain showed signs of involutive changes: the expansion of the subarachnoid space, the expansion of convexital sulci, ventricular enlargement, increased "transparency" of the white matter of the periventricular regions, multiple small calcifications in the projection of anterior cerebral arteries, focuses of demyelization of the cortical regions of the frontal lobes (Figure 7).

SG and REG revealed quite pronounced decrease in the rate of cerebral blood flow and pulse volume.

Cerebral MRA revealed multiple stenosis of intracranial branches (Figure 8).

The patient underwent a course of conservative treatment. A decrease in vestibular disorders was clinically observed. SG and REG showed some positive changes in cerebral blood flow and pulse volume velocity. In the subsequent period of conservative treatment, courses were held twice a year.

In 3 years, some positive changes are clinically preserved, there are no clear signs of dementia, and the severity of cognitive impairment is preserved at 27 MMSE points, the level of everyday life-100 IB points. SG and REG showed preserved positive dynamics. CT demonstrated further growing of involutive changes (Figure 9).

In another 3 years of conservative treatment courses, there were no signs of clinically positive dynamics, the level of dementia is equal to CDR-1, the severity of cognitive impairment equals 26 MMSE points, the level of everyday living-95 IB points. According to SG and REG, the positive trend will continue. CT noted further progression of cerebral involutive changes (Figure 10).

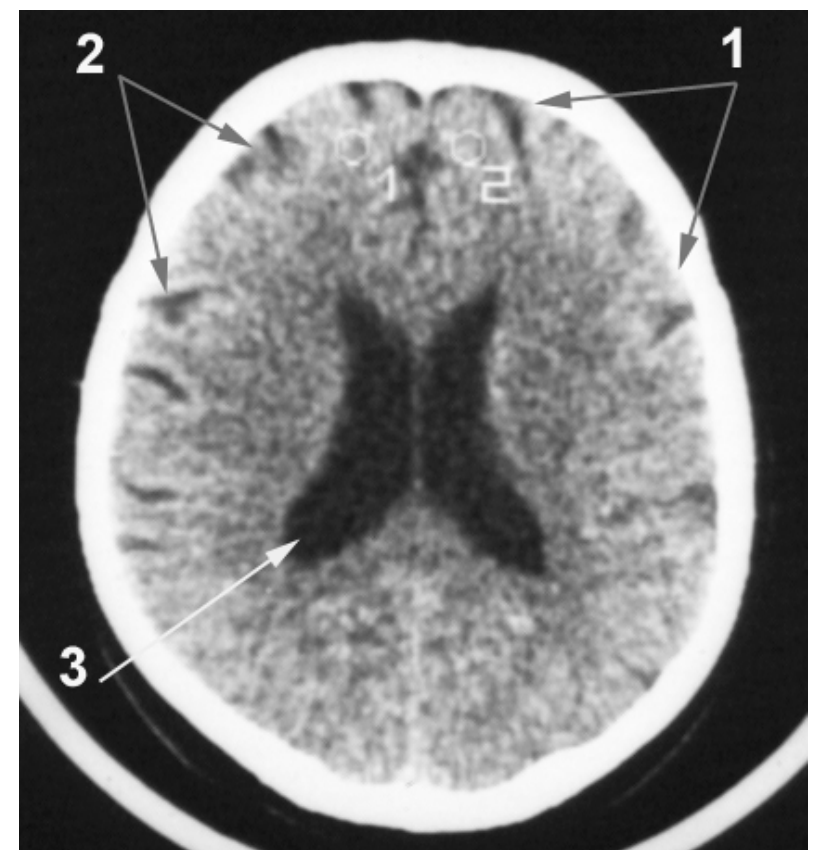

Figure 7. Patient K., male, 61 years old. CT of the brain. Before the treatment: 1) subarachnoid space expansion; 2) convexital sulci expansion; 3) ventricles enlargement. 


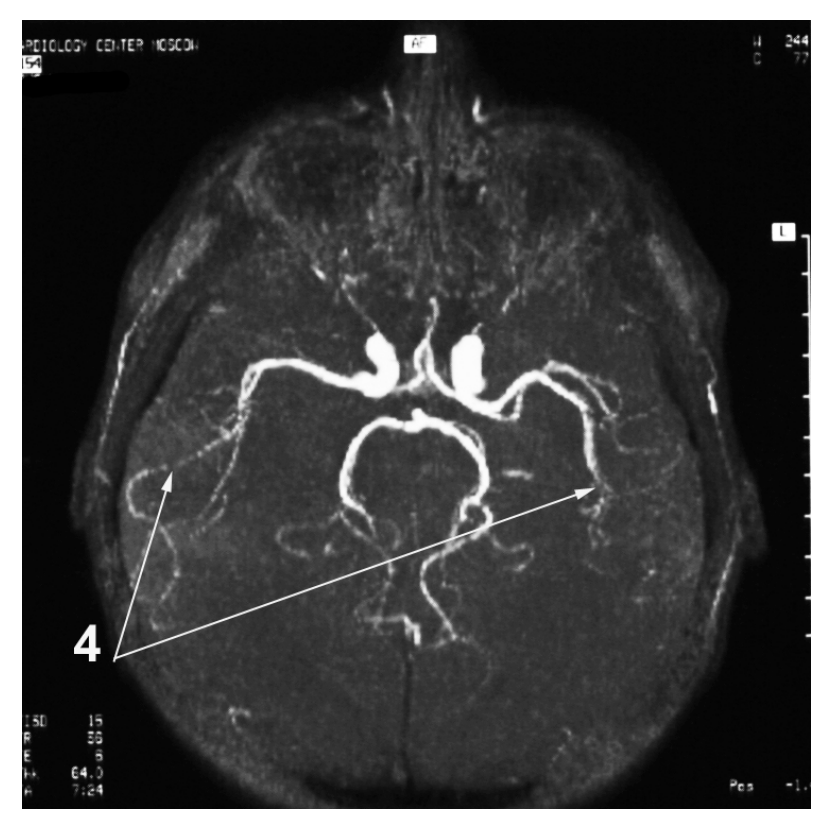

Figure 8. The same patient K.: MRA of the brain. 4) Multiple stenosis of intracranial branches.

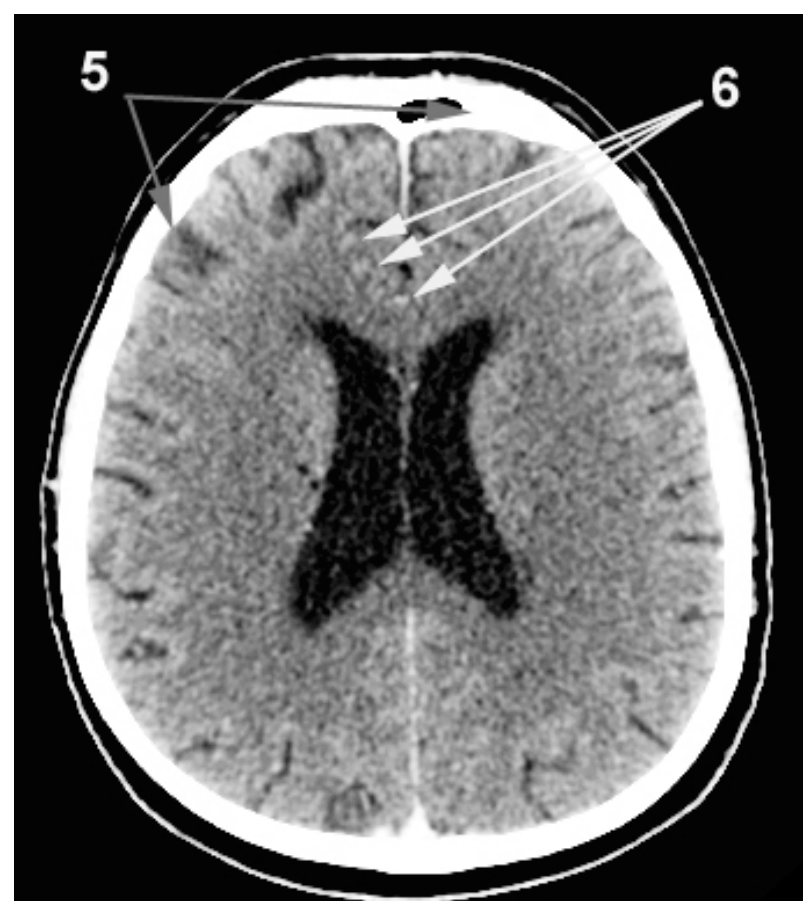

Figure 9. The same patient K.: CT of the brain. 3 years after the beginning of the treatment: 5) further growth of involutive changes; 6) multiple small calcifications in the projection of anterior cerebral arteries.

\section{Discussion}

Atherosclerosis is a systemic disease in which vascular lesions of the brain progress in various arterial basins, both in the right and in the left hemispheres. The process may evolve at a different speed, but always on two sides. Decreased blood flow stimulates angiogenesis, which is manifested in natural opening of collateral bed 


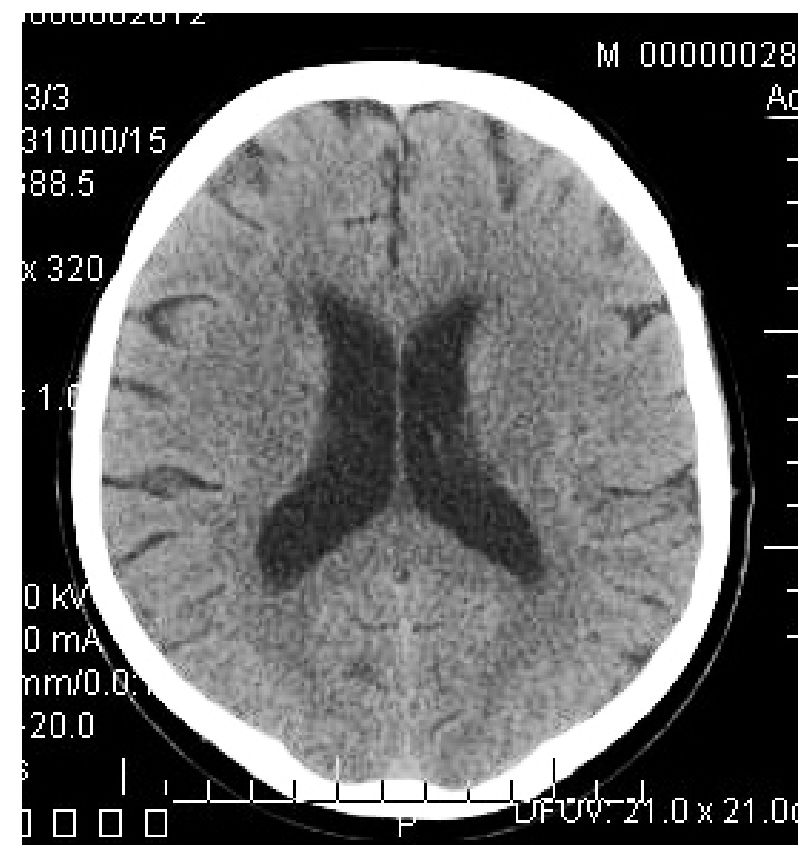

Figure 10. The same patient K.: CT of the brain. 6 years after the beginning of the treatment: further growth of involutive changes.

aimed at additional bypass blood flow to ischemic areas of the brain [2] [5] [9]. Unfortunately, angiogenesis is normally slow and is not always effective enough to fully restore the blood supply.

The two-sided nature of the lesion should be considered when carrying out any intervention, both on the brachiocephalic vessels and on the vessels of the brain [9]. Unilateral intervention is not always appropriate. For this reason, relying on MUGA data, we almost always performed the intervention both on one and on the other sides of the brain during laser revascularization. Naturally, improved blood circulation of the more affected hemisphere contributes to its functional recovery. Improved blood circulation of a less atherosclerosis-affected contralateral hemisphere also helps to improve its functionality, and at the same time, due to stimulation of collateral circulation, improves blood flow to the other hemisphere [2] [9] [34]. Thus, the two-way blood circulation improvement leads to an overall functional recovery of the brain.

Target high-energy laser irradiation allows destroying atherosclerotic tissues and restoring the patency and lumen of atherosclerotic vessels without affecting the vascular wall [9] [33] [34]. Resultant decomposition products of atherosclerotic tissues are of a very small size, 2 - 6 micrometers [32] [33]; they are naturally utilized, so they do not require any means of distal protection [34].

The mechanism of low-energy laser exposure lies in the powerful stimulation of natural physiological angiogenesis [2] [34] [35] causing collateral and capillary revascularization of tissues [2] [36]-[38]. At the same time, the deeply penetrating laser energy stimulates the metabolic processes in neurons increasing their energy resource through effects on mitochondria and providing neuroprotection [34]. These data are supported by the work of many authors who conducted both experimental and clinical studies dedicated to extracranial laser exposure of the brain [11]-[13] [15] [39] [40]. The after-treatment gradual increase in the mass of brain tissue suggests regenerative processes developing in it [34] [39] [41]. These data are also indirectly confirmed by experimental and clinical studies that prove the possibility of regenerative processes in the brain tissue [42].

Conservative therapies for atherosclerotic lesions of the brain fail to achieve pervasive and persistent cerebral revascularization. They only allow improving the blood supply to a certain extent. The resultant therapeutic effect is of short duration and is mainly associated with a partial improvement in metabolic processes, but this is often insufficient for the functional recovery of ischemic brain.

\section{Conclusions}

The method of transcatheter laser revascularization of cerebral vessels is a physiological, effective and low-in- 
vasive treatment for patients suffering from cerebral arteriosclerosis complicated by chronic cerebrovascular insufficiency. The method has virtually no alternatives; it can restore the patency and lumen of vessels of various diameter, while at the same time it stimulates natural angiogenesis, causes collateral and capillary revascularization both in the ischemic area and in the near tissues, promotes reparative processes in the brain tissue, which in turn promotes functional recovery of the brain.

The effect obtained after the treatment is maintained for a long time (up to 10 years or more); it causes regression of mental and motor disorders, promotes regression of dementia and can improve the patients' quality of life, which makes the proposed method significantly different from conservative treatment methods.

\section{References}

[1] Gillum, R.F., Kwagyan, J. and Obisesan, Th.O. (2011) Ethnic and Geographic Variation in Stroke Mortality Trends. Stroke, 42, 3294-3296. http://dx.doi.org/10.1161/STROKEAHA.111.625343

[2] Maksimovich, I.V. (2012) Transcatheter Treatment of Atherosclerotic Lesions of the Brain Complicated by Vascular Dementia Development. World Journal of Neuroscience, 2, 200-209. http://dx.doi.org/10.4236/wjns.2012.24031

[3] Frölich, A.M., Psychogios, N.M., Klotz, E., Knauth, M. and Knauth. P. (2012) Angiographic Reconstructions from Whole-Brain Perfusion CT for the Detection of Large Vessel Occlusion in Acute Stroke. Stroke, 43, 97-102. http://dx.doi.org/10.1161/STROKEAHA.111.630954

[4] Abou-Chebl, A. (2013) Management of Acute Ischemic Stroke. Current Cardiology Reports, 15, 348-354. http://dx.doi.org/10.1007/s11886-013-0348-4

[5] Gjulev, N.M., Pustozertsev, V.G. and Gjulev, S.N. (2002) Cerebrovascular Diseases. BINOM, Moscow. http://www.combook.ru/product/158808/

[6] Qureshi, A.I. and Caplan, L.R. (1014) Intracranial Atherosclerosis. Lancet, 15, 984-998. http://www.ncbi.nlm.nih.gov/pubmed/24007975

[7] Caplan, L.R., Thomas, A.J. and Inoa, V. (2014) Interventional Treatment of Brain Ischemia Related to Intracranial Cerebrovascular Occlusive Lesions. Current Opinion in Neurology, 27, 1-7. http://dx.doi.org/10.1097/WCO.0000000000000044

[8] Pendlebury, S.T., Wadling, S., Silver, L.E. and Rothwell, P.M. (2011) Transient Cognitive Impairment in TIA and Minor Stroke. Stroke, 42, 3116-3121. http://dx.doi.org/10.1161/STROKEAHA.111.621490

[9] Maksimovich, I.V. (2013) Possibilities of Transcatheter Treatment of Patients after Extensive Ischemic Stroke. World Journal of Neuroscience, 3, 171-185. http://dx.doi.org/10.4236/wjns.2013.33022

[10] Klopfenstein, J.D., Ponce, F.A., Kim, L.J., Albuquerque, F.C., Nakaji, P. and Spetzler, R.F. (2005) Middle Cerebral Arterystenosis: Endovascular and Surgicaloptions. Skull Base, 15, 175-189. http://dx.doi.org/10.1055/s-2005-871873

[11] Hashmi, J.T., Huang, Y.-Y., Osmani, B.Z., et al. (2010) Role of Low-Level Laser Therapy in Neurorehabilitation. PM \& R, 2, S292-S305. http://www.ncbi.nlm.nih.gov/pmc/articles/PMC3065857/

[12] Naeser, M.A. and Hamblin, M.R. (2011) Potential for Transcraniallaser or LED Therapy to Treat Stroke, Traumatic Brain Injury, and Neurodegenerative Disease. Photomedicine and Laser Surgery, 29, 443-446.

http://www.ncbi.nlm.nih.gov/pubmed/21728786 http://dx.doi.org/10.1089/pho.2011.9908

[13] Song, S., Zhou, F. and Chen, W.R. (2012) Low-Level Laser Therapy Regulates Microglial Function through Src-Mediated Signaling Pathways: Implications for Neurodegenerative Diseases. Journal of Neuroinflammation, 9, 219. http://www.ncbi.nlm.nih.gov/pubmed/22989325

[14] Stephan, W., Banas, L.J., Bennett, M. and Tunceroglu, H. (2012) Efficacy of Super-Pulsed 905 NM Low Level Laser Therapy (LLLT) in the Management of Traumatic Brain Injury (TBI): A Case Study. World Journal of Neuroscience, 2, 231-233. http://www.scirp.org/journal/PaperInformation.aspx?paperID=24793\&\#abstract

[15] Konstantinović, L.M., Jelić, M.B., Jeremić, A., Stevanović, V.B., Milanović, S.D. and Filipović, S.R. (2013) Transcranial Application of Near-Infrared Low-Level Laser Can Modulate Cortical Excitability. Lasers in Surgery and Medicine, 45, 648-653. http://www.ncbi.nlm.nih.gov/pubmed/24136303 http://dx.doi.org/10.1002/lsm.22190

[16] Takaiwa, A., Kuwayama, N., Akioka, N., Kurosaki, K., et al. (2013) Effect of Carotid Endarterectomy on Cognitive Function in Patients with Asymptomatic Carotid Artery Stenosis. Acta Neurochirurgica, 155, 627-633. http://link.springer.com/article/10.1007/s00701-013-1625-0 http://dx.doi.org/10.1007/s00701-013-1625-0

[17] Altinbas, A., Algra, A., Martin, M., Brown, M.M., et al. (2014) Effects of Carotid Endarterectomy or Stenting on He- 
modynamic Complications in the International Carotid Stenting Study: A Randomized Comparison. International Journal of Stroke, 9, 284-290. http://onlinelibrary.wiley.com/enhanced/doi/10.1111/ijs.12089/

[18] Muroi, C., Khan, N., Bellut, D., Fujioka, M. and Yonekawa, Y. (2011) Extracranial-Intracranial Bypass in Atherosclerotic Cerebrovascular Disease: Report of a Single Centre Experience. British Journal of Neurosurgery, 25, 357-362. http://www.ncbi.nlm.nih.gov/pubmed/21501047 http://dx.doi.org/10.3109/02688697.2010.551673

[19] Papanagiotou, P., Roth, C., Walter, S., Behnke, S., Grunwald, L.Q., Viera, J., Politi, M., Körner, H., Kostopoulos, P., Haass, A., Fassbender, K. and Reith, W. (2011) Carotid Artery Stenting in Acute Stroke. Journal of the American College of Cardiology, 58, 2363-2369. http://content.onlinejacc.org/article.aspx?articleid=1147802 http://dx.doi.org/10.1016/j.jacc.2011.08.044

[20] Matsumaru, Y., Tsuruta, W., Takigawa, T., Hyodo, A., Sato, H. and Matsumura, A. (2004) Percutaneous Transluminal Angioplasty for Atherosclerotic Stenoses of Intracranial Vessels. Interventional Neuroradiology, 10, 17-20. http://www.ncbi.nlm.nih.gov/pmc/articles/PMC3522214/

[21] Derdeyn, C.P. and Chimowitz, M.I. (2007) Angioplasty and Stenting for Atherosclerotic Intracranial Stenosis: Rationale for a Randomized Clinical Trial. Neuroimaging Clinics of North America, 17, 355-ix. http://www.ncbi.nlm.nih.gov/pmc/articles/PMC2040119/

[22] Dorn, F., Prothmann, S., Wunderlich, S. and Liebig, T. (2012) Stent Angioplasty of Intracranial Stenosis: Single Center Experience of 54 Cases. Clinical Neuroradiology, 22, 149-156.

http://www.ncbi.nlm.nih.gov/pubmed/?term=Dorn+F\%2C+Prothmann+S\%2C+Wunderlich+S\%2C+Liebig+T http://dx.doi.org/10.1007/s00062-011-0106-1

[23] El Ali, A., Doeppner, T.R. and Hermann, D.M. (2011) Increased Blood-Brain Barrier Permeability and Brain Edema after Focal Cerebral Ischemia Induced by Hyperlipidemia: Role of Lipid Peroxidation and Calpain-1/2, Matrix Metalloproteinase-2/9, and RhoA Overactivation. Stroke, 42, 3238-3244.

http://www.ncbi.nlm.nih.gov/pubmed/21836084 http://dx.doi.org/10.1161/STROKEAHA.111.615559

[24] Moustafa, R.R., Momjian-Mayor, I., Simon Jones, P., Morbelli, S., Day, D.J., Aigbirhio, F.I., Fryer, T.D., Warburton, E.A. and Baron, J.C. (2011) Microembolism versus Hemodynamic Impairment in Rosary-Like Deep Watershed Infarcts: A Combined Positron Emission Tomography and Transcranial Doppler Study. Stroke, 42, 3138-3143. http://stroke.ahajournals.org/content/42/11/3138.abstract http://dx.doi.org/10.1161/STROKEAHA.111.616334

[25] Maksimovich, I.V. (2006) Method for Carrying out Transluminal Laser-Induced Brain Revascularization in Atherosclerotic Injury Cases. RF Patent No. 2297861.

http://worldwide.espacenet.com/publicationDetails/biblio?II=3\&ND=3\&adjacent=true\&locale=en EP\&FT=D\&date=2 $0070427 \& C C=\mathrm{RU} \& \mathrm{NR}=2297861 \mathrm{C} 1 \& \mathrm{KC}=\mathrm{C} 1$

[26] Maksimovich, I.V. (2006) Method of Transluminal Laser Revascularization of Cerebral Blood Vessels Having Atherosclerotic Lesions. US Patent No. 7490612.

http://www.patentlens.net/patentlens/patents.html?patnums=US_7490612\&returnTo=patentnumber.html\%3Fquery\%3 D\%2528US_7490612\%2Bin\%2Bpublication_number\%2529

[27] Morris, J.C. (1993) The Clinical Dementia Rating (CDR): Current Version and Scoring Rules. Neurology, 43, 24122414. http://www.neurology.org/content/43/11/2412.2 http://dx.doi.org/10.1212/WNL.43.11.2412-a

[28] Folstein, M.F., Folstein, S.E. and McHugh, P.R. (1975) "Mini-Mental State”: A Practical Method for Grading the Cognitive State of Patients for the Clinician. Journal of Psychiatric Research, 12, 189-198. http://www.journalofpsychiatricresearch.com/article/0022-3956(75)90026-6/abstract http://dx.doi.org/10.1016/0022-3956(75)90026-6

[29] Mahoney, F.I. and Barthel, D.M. (1965) Functional Evaluation: The Barthel Index. Maryland State Medical Journal, 14, 61-65. http://www.ncbi.nlm.nih.gov/pubmed/14258950

[30] Maksimovich, I.V. (2013) Transcatheter Treatment of Patients after Extensive Ischemic Stroke. Journal of the American College of Cardiology, 62, B155-B156. http://content.onlinejacc.org/article.aspx?articleid=1759823

[31] Deviatkov, N.D., Rabkin, I.Kh., Maksimovich, I.V., Rogov, R.A. and Aleinikov, V.S. (1986) Use of Copper-Vapor Laser Radiation for the Evaporation of Atherosclerotic Lesions of the Major Arteries in Vitro. Surgery, 4, 116-121. http://www.ncbi.nlm.nih.gov/pubmed/3713043

[32] Petrovskiy, B.V., Deviatkov, N.D., Rabkin, I.Kh., Maksimovich, I.V. and Rogov, K.A. (1986) Destruction of Arteriosclerotic Lesions of Human Cadaveric Arteries by Laser Irradiation with Copper Vapors. Surgery, 5, 112-116. http://www.ncbi.nlm.nih.gov/pubmed/3723942

[33] Kuleshov, E.V. and Maksimovich, I.V. (1994) Endovascular Surgery in Patients over 65 with Disseminated Atheros- 
clerosis of the Vessels of the Pelvis and Lower Extremities. Vestnik Surgery Imeni I. I. Grekova, 152, 27-30. http://www.ncbi.nlm.nih.gov/pubmed/7701734

[34] Maksimovich, I.V. (2004) Transljuminal Laser Angioplasty in Treatment of Ischemic Lesions of a Brain. M.D. Dissertation, Russian University of Friendship of the People, Moscow. http://disseng.com/page/order/id/206426.html

[35] Chizhov, G.K., Kovalskaia, N.I. and Kozlov, V.I. (1991) The Effect of Helium-Neon Laser Radiation on the Energy Metabolic Indices of the Myocardium. Bulletin of Experimental Biology and Medicine, 111, 302-305. http://www.ncbi.nlm.nih.gov/pubmed/2054512 http://dx.doi.org/10.1007/BF00840904

[36] Kozlov, V.I. and Azizov, G.A. (2007) Pathophysiological Characteristics of Microcirculatory Disorders in Chronic Arterial Ischaemia of Lower Limbs. Angiology and Vascular Surgery, 13, 17-23. http://www.ncbi.nlm.nih.gov/pubmed/17679971

[37] Moskvin, S.V. (2008) System Analysis of Efficiency in Controlling Biological Systems with Low-Energy Laser Radiation. Doctor Thesis, Tula. http://www.dissers.info/disser_323120.html

[38] Mak, M.C. and Cheing, G.L. (2012) Immediate Effects of Monochromatic Infrared Energy on Microcirculation in Healthy Subjects. Photomedicine and Laser Surgery, 30, 193-199. http://europepmc.org/abstract/MED/22220935 http://dx.doi.org/10.1089/pho.2011.3012

[39] Barrett, D.W. and Gonzalez-Lima, F. (2013) Transcranial Infrared Laser Stimulation Produces Beneficial Cognitive and Emotional Effects in Humans. Neuroscience, 230, 13-23. http://www.ncbi.nlm.nih.gov/pubmed/23200785 http://dx.doi.org/10.1016/j.neuroscience.2012.11.016

[40] Yang, X., Askarova, S., Sheng, W., et al. (2010) Low Energy Laser Light (632.8 NM) Suppresses Amyloid- $\beta$ Peptide-Induced Oxidative and Inflammatory Responses in Astrocytes. Neuroscience, 171, 859-868. http://www.sciencedirect.com/science/article/pii/S030645221001273X?np=y

[41] Starck, T., Nissilä, J., Aunio, A., Abou-Elseoud, A., Remes, J., Nikkinen, J., Timonen, M., Takala, T., Tervonen, O. and Kiviniemi, V. (2012) Stimulating Brain Tissue with Bright Light Alters Functional Connectivity in Brain at the Resting State. World Journal of Neuroscience, 2, 81-90. http://www.scirp.org/journal/PaperInformation.aspx?paperID=19417\&\#abstract

[42] Heinrich, C., Blum, R., Gascón, S., et al. (2010) Directing Astroglia from the Cerebral Cortex into Subtype Specific Functional Neurons. PLoS Biology, 8, e1000373. http://www.ncbi.nlm.nih.gov/pubmed/20502524 http://dx.doi.org/10.1371/journal.pbio.1000373 
Scientific Research Publishing (SCIRP) is one of the largest Open Access journal publishers. It is currently publishing more than 200 open access, online, peer-reviewed journals covering a wide range of academic disciplines. SCIRP serves the worldwide academic communities and contributes to the progress and application of science with its publication.

Other selected journals from SCIRP are listed as below. Submit your manuscript to us via either submit@scirp.org or Online Submission Portal.
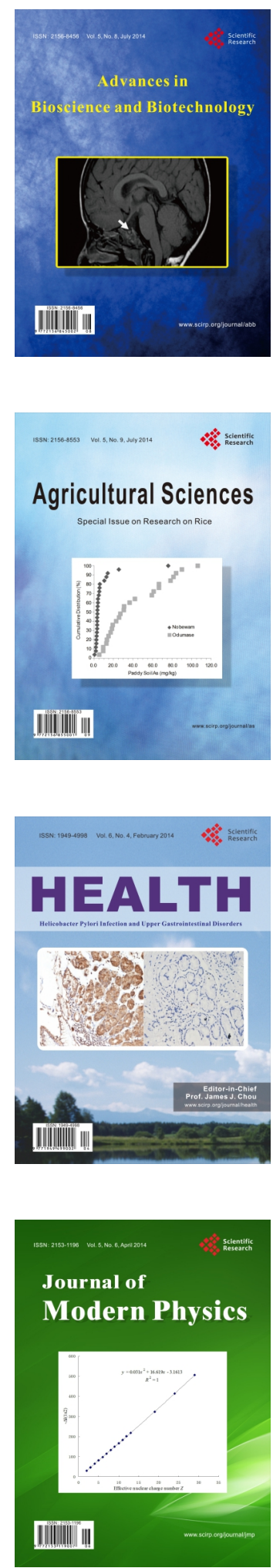
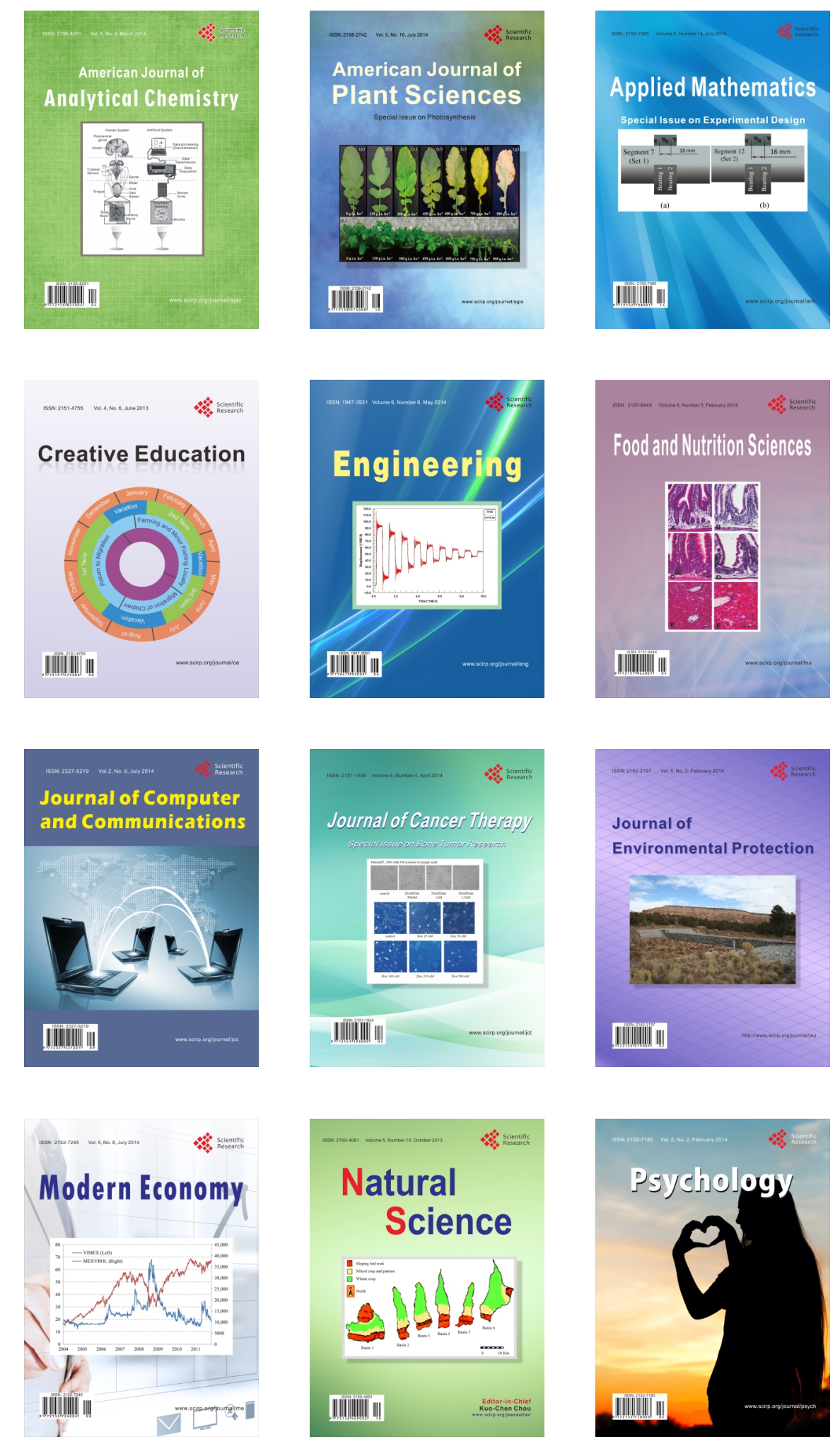